\title{
Epidemiological and Clinical Characteristics of COVID-19 in Indian Children in the Initial Phase of the Pandemic
}

\author{
Bhakti Sarangi, Venkat Sandeep Reddy, Jitendra S Oswal, Nandini Malshe, AJinkya Patil, Manojit \\ Chakraborty and SANJAY LalWani \\ From Department of Pediatrics, Bharati Vidyapeeth Medical College and Hospital, Pune, Maharashtra, India.
}

\author{
Correspondence to: Jitendra S Oswal, \\ Professor, Department of Pediatrics, \\ Bharati Vidyapeeth Medical College \\ and Hospital, Pune 411043, \\ Maharashtra, India. \\ jsoswal@gmail.com \\ Received: June 08, 2020; \\ Initial review: June 19, 2020; \\ Accepted: July 28, 2020.
}

\begin{abstract}
Objective: To assess the epidemiological and clinical characteristics of pediatric inpatients with COVID-19, early in the pandemic. Methods: Clinical and laboratory profile and outcomes were studied for children (aged 1 month - 18 years) presenting between 1 April, 2020 and 20 May, 2020 with positive nasopharyngeal swab for SARS-CoV-2 by RT-PCR. Results: 50 children (56\% male) with median (IQR) age of $6(2-12)$ years were included. Majority $(56 \%)$ were from families belonging to Kuppuswamy upper lower socioeconomic class. $45(90 \%)$ had positive household contact, and $33(66 \%)$ had overcrowding at home. $29(58 \%)$ children were asymptomatic while 20 (40\%) had mild symptoms. Fever, cough, and sore throat were the most common symptoms. High C-reactive protein levels were seen in $15(30 \%)$ children. There was no mortality. Conclusion: The disease burden appears high in lower socio-economic group with majority having a positive household contact. Milder disease pattern in the pediatric age group is reiterated.
\end{abstract}

Keywords: Management, RT-PCR, SARS-CoV-2, Symptoms, Outcome.

Published online: July 28, 2020; PII: S097475591600218

$\longrightarrow$ oronavirus disease 2019 (COVID-19), caused by severe acute respiratory syndrome coronavirus 2 (SARS-CoV-2), has been in circulation for more than six months now [1]. Though there have been a growing number of studies focused on COVID-19, limited data is available on epidemiological features, clinical manifestations, and transmission patterns in children with COVID-19, more so from India. Early observations in a pandemic are pivotal in improving the understanding of the physiological patterns and varied clinical profiles, so as to improve early recognition and appropriate management. We, therefore, describe the clinical and epidemiological features of pediatric patients seen at a single tertiary-care institution.

\section{METHODS}

This was a cross-sectional study conducted in a dedicated pediatric COVID-19 center in Pune, Maharashtra between 1 April, 2020 and 20 May, 2020. Prior approval was taken from the institutional ethics committee. All children between one month and 18 years of age who tested positive by the RT- PCR technique for nasopharyngeal swab were included in the study - these also included asymptomatic children as per the management guidelines in force. Written informed consent was taken from the parents of all children and assent was taken from children who were greater than 9 years of age. Detailed information including demographic data, travel and contact history, living conditions and overcrowding, symptoms, and presence of co-morbid conditions were taken. The children were examined and categorized as per degree of severity based on standard criteria [2].

Baseline laboratory parameters (complete hemogram and C-reactive protein) were evaluated and repeated as required. Chest radiograph was done in all symptomatic children. On chest $X$-ray each lung was divided into three zones. Each zone was given a score of 1 if there was any opacity and 0 if there were none. Total score of 3 was considered as 50\% involvement [3]. All children admitted were managed as per the hospital protocol. The children were monitored daily for changes in disease severity. Discharge from hospital was as per prescribed World Health Organization (WHO) guidelines which stated that asymptomatic children who tested negative for two nasopharyngeal swabs taken 24 hours apart after day 14 of illness were fit for discharge [4]. Overcrowding was defined based on persons per room criteria [5].

Statistical analyses: The data were analyzed using the Statistical Package for Social Sciences (SPSS) software version 25.0. Spearman's Rho correlation coefficient was used to determine the correlation with disease severity. A $P$ value $<0.05$ was considered significant. 


\section{RESULTS}

A total of 178 children presented to us with suggestive features during the study duration, of which, 153 were negative and 25 were positive for SARS-CoV-2 by RTPCR. Another 25 children with a positive RT-PCR were referred from other hospitals. Thus, a total of 50 children (56\% males) with median (IQR) age of 6 (2-12) years were included. Majority $(82 \%)$ of the cases hailed from containment zones in Pune. There was history of positive household contact in $45(90 \%)$ children; with 42 having family members with mild illness and three with severe illness. Travel history to affected area was documented in only one child (Table I).

More than half $(58 \%)$ of the children were asymptomatic while $20(40 \%)$ had mild symptoms. In symptomatic children, fever was the chief complaint in 17 (34\%). None of the children had hypoxemia measured by pulse oximetry. Only two children had co-morbidities; one child had history of simple febrile seizures, and another had underlying type I diabetes mellitus and had presented with diabetic ketoacidosis. Immunization was complete in $32(64 \%)$ of the children as per universal immunization program, and $49(98 \%)$ children had a BCG scar.

The mean (SD) leucocyte count was 8864 (3727.2) X10 $9 /$ L (range, 3300-19300 X10 $\% / L$ ). Leucopenia was seen in $3(6 \%)$ children while leukocytosis was seen in $13(26 \%)$ children. Lymphopenia, eosinopenia and thrombocytopenia were not seen in any child. Neutrophillymphocyte-ratio (NLR) $(r=0.35, P=0.01)$ and lymphocytemonocyte-ratio (LMR) $(r=-0.31, P=0.03)$ showed a significant correlation with the severity of the illness, while platelet-lymphocyte ratio (PLR) $(r=0.28, P=0.06)$ and CRP $(r=0.05, P=0.73)$ did not show any correlation with severity of the disease.

Chest radiograph was done in $20(95.2 \%)$ of 21 symptomatic children. It was found to be normal in 18 $(85.7 \%)$, while two showed bilateral lower zone haziness $(<50 \%)$. The disease category for all patients remained same all through the hospital stay and no mortality was seen.

\section{DISCUSSION}

Majority of children in our study were detected in the identified containment zones, most of them reporting exposure to a positive household contact. Majority of the children were either asymptomatic or had mild disease.

Most children were from lower socio-economic groups, a pattern also witnessed in other countries [6]. Though the disease was seen in all age groups, children less than five years of age accounted for nearly half the cases. This can be attributed to the inability of this age group to comprehend and follow social distancing norms and their frequent close contact with parents.

Pediatric observational studies published early in the spread across China reported similar clinical findings with fever being the most common symptom followed by cough and sore throat [7]. A recent meta-analysis has also shown that most of the patients have mild to moderate disease $(96 \%)$ with only $1 \%$ of all the symptomatic pediatric cases being critically sick [8]. The reported mortality rate of COVID-19 in children is less than 1\% [9]. Various hypotheses have been proposed for the lesser disease severity in children [10], though a definite answer is still awaited.

Table I Epidemiological and Clinical Characteristics of Children With SARS-CoV-2 Infection in Pune, 2020 ( $N=50)$

\begin{tabular}{|c|c|}
\hline Parameters & No. $(\%)$ \\
\hline Male & $28(56)$ \\
\hline \multicolumn{2}{|l|}{ Age } \\
\hline $1 \mathrm{mo}$ to $1 \mathrm{y}$ & $9(18)$ \\
\hline$>1$ to $5 \mathrm{y}$ & $15(30)$ \\
\hline$>5$ to $10 \mathrm{y}$ & $12(24)$ \\
\hline$>10$ to $15 \mathrm{y}$ & $11(22)$ \\
\hline$>15$ to $18 \mathrm{y}$ & $3(6)$ \\
\hline Weight-for-age (3-97centile) & $45(90)$ \\
\hline Overcrowding & $33(66)$ \\
\hline Contact with patient of COVID- 19 & $45(90)$ \\
\hline \multicolumn{2}{|l|}{ Socio-economic status* } \\
\hline Upper lower & $28(56)$ \\
\hline Lower middle & $17(54)$ \\
\hline Upper middle & $5(10)$ \\
\hline \multicolumn{2}{|l|}{ Severity of illness } \\
\hline Asymptomatic & $29(58)$ \\
\hline Mild & $20(40)$ \\
\hline Moderate & $1(2)$ \\
\hline Severe & $0(0)$ \\
\hline \multicolumn{2}{|l|}{ Symptoms } \\
\hline Fever & $17(34)$ \\
\hline Cough & $8(16)$ \\
\hline Sore throat & $7(14)$ \\
\hline Myalgia & $4(8)$ \\
\hline Diarrhea & $2(4)$ \\
\hline Headache & $2(4)$ \\
\hline
\end{tabular}




\section{WHAT THIS STUDY ADDS?}

- Majority of Indian children with SARS-CoV-2 infection had a mild course of disease during the initial stages of the pandemic.

Malnutrition has been deemed a risk factor in adult COVID-19 [11]. In children, malnutrition is known to foster infections; however, in this study, majority of the children were well-nourished as per weight-for-age criteria. The hematological profile of adults with COVID-19 has demonstrated leucopenia with associated neutrophilia, lymphopenia; eosinopenia and thrombocytopenia. Also, higher NLR, LMR and PLR have been associated with severe disease and used for prognostication [12]. Leucopenia, however, was seen in only $6 \%$ of our children and there was no evidence of lymphopenia, thrombocytopenia or eosinopenia. Increasing NLR in our study showed a moderate positive correlation coefficient while LMR showed a negative correlation. High CRP values have now become synonymous with severe COVID-19 infection among adults as seen in majority of the studies [13]. The value of CRP did not correlate with disease severity in our study. These discordant results may be due to the majority of our patients being asymptomatic or mildly symptomatic, or due to a different history of antigen exposure and immune response.

Repeat RT-PCR of nasopharyngeal swab was done on day 14 and 15 to check for infectivity status. All the children except one tested negative by RT-PCR on both the days. For the child who tested positive for one swab, a repeat swab was negative after three days, thus indicating that clearance of viral load may vary in different individuals. The degree of infectivity of these individuals after 14 days remains questionable as RT-PCR detects genetic fragments of the virus and cannot distinguish between dead or live virus [14]. In such scenarios, doing a viral culture may be the plausible method of detecting live virus and demonstrating continued infectivity. As performing a viral culture is difficult and requires advanced laboratory facilities, using GeneXpert platform with (cycle threshold) $C t$ values $\geq 24$ may also be beneficial for predicting lack of infectivity [15].

The findings of our study are limited by the size of the cohort and may require further validation by a study with a larger sample size. Being a study in the initial phase of the pandemic with lockdown in place, it may not cover the entire spectrum of clinical presentations, severity and magnitude of SARS-CoV-2 in children from different geographical areas. We could also not collect data for calculation of body mass index (BMI) and Z-scores.
Table II Laboratory Investigations of Children With SARSCoV-2 Infection in Pune, $2020(N=50)$

\begin{tabular}{lr}
\hline Parameter & Value \\
\hline Absolute neutrophil count $\left(\times 10^{9} / \mathrm{L}\right)$ & $2480(1995.5-3339)$ \\
Absolute lymphocyte count $\left(\times 10^{9} / \mathrm{L}\right)$ & $4071(2912-5964)$ \\
Absolute monocyte count $\left(\times 10^{9} / \mathrm{L}\right)$ & $576(402.5-744)$ \\
Absolute eosinophil count $\left(\times 10^{9} / \mathrm{L}\right)$ & $156(68.5-437.5)$ \\
Leucopenia* & $3(6)$ \\
High C-reactive protein* & $15(30)$ \\
\hline
\end{tabular}

All values in median (IQR) except *no.(\%); leucopenia-leucocyte count $<4000 \times 10^{9} / \mathrm{L}$; High C-reactive protein-value $>6 \mathrm{mg} / \mathrm{dL}$.

In conclusion, our study shows that there is a higher disease burden in lower-socioeconomic groups with majority of children having a positive household contact. A milder disease pattern is seen in majority of children with COVID-19.

Ethical approval: Institutional Ethics Committee of Bharati Vidyapeeth Medical College and Hospital; No. BVUDMC/IEC/ 1B, dated 10 April, 2020.

Contributors: VSR,BS,AP,MC: management of the patients; VSR,BS: collected the data, reviewed the literature and drafted the first version of the manuscript; BS,JSO,NM,SL: conceptualized the study, reviewed the literature, revised the manuscript and critically reviewed the manuscript. All authors contributed to manuscript preparation and approved the final version of the manuscript.

Funding: None; Competing interests: None stated.

\section{REFERENCES}

1. Riou J, Althaus CL. Pattern of early human-to-human transmission of Wuhan 2019 novel coronavirus (2019nCoV), December 2019 to January 2020. Euro Surveill. 2020;25:2000058

2. Guidelines On Clinical Management Of COVID-19. Government of India Ministry of Health \& Family Welfare Directorate General of Health Services (EMR Division), pp.3-5. Available at: https://www.mohfw.gov.in/pdf/ GuidelinesonClinicalManagementofCOVID 1912020.pdf. Accessed July 27, 2020.

3. Toussie D, Voutsinas N, Finkelstein M, Cedillo M, Manna $\mathrm{S}$, Maron S, et al. Clinical and chest radiography features determine patient outcomes in young and middle age adults with COVID-19. Radiol. 2020;201754.

4. Global Surveillance for human infection with novel coronavirus(2019-nCoV). Available from: https:// 
www.who.int/publications-detail/global-surveillance-forhuman-infection-with-novel-coronavirus-(2019-ncov). Accessed June 20, 2020

5. Park K. Environment and Health. In: Park K, ed. Park's Textbook of Preventive and Social Medicine. 23rd ed. Jabalpur: Bhanot Publishers; 2015.p.758.

6. Coronavirus Disease 2019 (COVID-19). Centers for Disease Control and Prevention. 2020. Available from: https://www.cdc.gov/coronavirus/2019-ncov/need-extraprecautions/racial-ethnic-minorities.html. Accessed June 20, 2020.

7. Qiu H, Wu J, Hong L, Luo Y, Song Q, Chen D. Clinical and epidemiological features of 36 children with coronavirus disease 2019 (COVID-19) in Zhejiang, China: An observational cohort study. Lancet Infect Dis. 2020;20:689-96.

8. Meena J, Yadav J, Saini L, Yadav A, Kumar J. Clinical features and outcome of SARS-CoV-2 infection in children: A systematic review and meta-analysis. Indian Pediatr. 2020 Jun 24. S097475591600203 [Epub ahead of print].

9. COVID-19: Data Summary - NYC Health. Available from: https://www1.nyc.gov/site/doh/covid/covid-19-data.page. Accessed June 20, 2020
10. Hoffmann M, Kleine-Weber H, Schroeder S, Krüger N, Herrler T, Erichsen S, et al. SARS-CoV-2 cell entry depends on ACE2 and TMPRSS2 and is blocked by a clinically proven protease inhibitor. Cell. 2020;181:271-80.e8

11. Li T, Zhang Y, Gong C, Wang J, Liu B, Shi L, et al. Prevalence of malnutrition and analysis of related factors in elderly patients with COVID-19 in Wuhan, China. Eur J Clin Nutr. 2020;74:871-9.

12. Lagunas-Rangel FA. Neutrophil-to-lymphocyte ratio and lymphocyte-to-C-reactive protein ratio in patients with severe coronavirus disease 2019 (COVID-19): A metaanalysis. J Med Virol. 2020;10.1002/jmv.25819 [published online ahead of print, 2020 Apr 3].

13. Wang L. C-reactive protein levels in the early stage of COVID-19. Med Mal Infect. 2020;50:332-4.

14. Lauri A, Mariani PO. Potentials and limitations of molecular diagnostic methods in food safety. Genes Nutr. 2008;4:1-12.

15. Bullard J, Dust K, Funk D, Strong JE, Alexander D, Garnett $\mathrm{L}$, et al. Predicting infectious SARS-CoV-2 from diagnostic samples. Clin Infect Dis. 2020; ciaa638 [published online ahead of print, 2020 May 22]. 